\title{
ANIMAL MODELS OF MATERNAL NUTRITION AND ALTERED OFFSPRING BONE STRUCTURE - BONE DEVELOPMENT ACROSS THE LIFECOURSE
}

\author{
Stuart A. Lanham ${ }^{1, *}$, Caroline Bertram ${ }^{1}$, Cyrus Cooper ${ }^{1,2}$ and Richard O.C. Oreffo ${ }^{1, *}$ \\ ${ }^{1}$ Bone and Joint Research Group, Human Development and Health, Institute of Developmental Sciences, \\ University of Southampton School of Medicine, Southampton, SO16 6YD, U.K. \\ ${ }^{2}$ MRC Lifecourse Epidemiology Unit, Southampton General Hospital, Southampton, SO16 6YD.
}

\begin{abstract}
It is widely accepted that the likelihood of offspring developing heart disease, stroke, or diabetes in later life, is influenced by the their in utero environment and maternal nutrition. There is increasing epidemiological evidence that osteoporosis in the offspring may also be influenced by the mother's nutrition during pregnancy. This review provides evidence from a range of animal models that supports the epidemiological data; suggesting that lifelong bone development and growth in offspring is determined during gestation.
\end{abstract}

Keywords: Animal models, bone, bone development, epidemiology, epigenetics.

*Address for correspondence:

S.A. Lanham

Bone and Joint Research Group

Human Development and Health

Institute of Developmental Sciences

University of Southampton School of Medicine

Southampton, SO16 6YD, U.K.

Or R.O.C. Oreffo

E-mail:sal3@soton.ac.uk

Address as above

E-mail: roco@soton.ac.uk

\section{Introduction}

Epidemiological evidence accrued over the last decade indicate that the environment and nutritional status experienced during in utero, postnatal as well as early life plays an important role in the aetiology of diseases such as osteoporosis (Cooper et al., 1997; Cooper et al., 2002; Jordan and Cooper, 2002). This environmental phenomenon often referred to as "programming", describes long-lasting changes in structure and function caused by environmental stimuli acting at critical periods during development. This concept, first detailed by Barker (Barker, 1992; Hales and Barker, 1992), has been informed by epidemiological studies on birth and long-term outcomes in diverse communities around the world, and by laboratory-based research looking at both specific systems and at the basic science underpinning developmental origins. Much of the work, until now, has been directed at cardiovascular and metabolic outcomes (Ozaki et al., 2001; Gluckman and Hanson, 2004; Gluckman et al., 2005; Gluckman and Hanson, 2006), however evidence is emerging that bone development and growth are programmed, although, interestingly, bone is constantly remodelled throughout life (Baird et al., 2011). In particular, maternal nutrition appears to be important in determining skeletal size at maturity. However, to date, there remains a paucity of understanding surrounding the cellular and, critically, molecular mechanisms whereby environmental modulation in utero results in an altered skeletal development among the offspring. This review will cover the emerging data from various animal models that have been used in the elucidation of the developmental origins of bone development across the lifecourse with correlation as available to the human scenario.

\section{Nutrition and Bone Development - Linking Cause and Effect}

It has been known for several decades that nutrition can permanently alter growth. Furthermore, there is increasing evidence that the timing of a nutritional insult can produce differing effects on skeletal development. Widdowson and McCance (Widdowson and McCance, 1963) demonstrated that if rats were undernourished 3-6 weeks after birth, the animals lost weight compared to control animals. These nutritionally challenged animals remained smaller, even when fed control levels of food. However, when rats 9-12 weeks of age were undernourished, these animals initially lost weight, but surprisingly regained their normal growth trajectory when returned to a normal diet; so-called 'catch 
up growth', indeed, their weight gain was observed to exceed the weight of the control group.

Nevertheless, it is not simply a lack of food that can alter growth. Various groups have shown that rats fed a low protein diet from weaning grew for a longer period (Reichling and German, 2000; Miller and German, 1999), but at a slower rate than control animals, and finally attained the same size as control animals. These studies demonstrate an important point that final mass at maturity can be programmed in utero, as post-weaning protein restriction does not affect the eventually size of the animal. Hence, there would appear to be a post-natal period where bone development (as assessed by final size and mass) is susceptible to nutritional challenge. Clinically, a long established example of such nutritional influences on bone development is Rickets, where incomplete mineralisation of the organic bone matrix arises as a consequence of poor childhood nutrition. This provides a well-established and documented example of how a post-natal period of nutrition can influence bone development. However, what is not well-established is the influence of the pre-natal maternal nutrition on bone development of the offspring.

Peak bone mass attained at skeletal maturity is a major risk factor for fracture in later life. The evidence to date suggests peak bone mass is partly inherited, however genetic markers only partially explain the differences in an individual's bone mass or fracture risk (Ralston, 1998). Epidemiological studies suggest impaired growth during fetal life, infancy and early childhood is associated with reduced adult bone mass (Cooper et al., 1995; Cooper et al., 1997; Fall et al., 1998; Cooper et al., 2002).

It is known that the longitudinal growth of long bones depends on a functional growth plate. Skeletal growth is regulated systemically by growth hormone with important contributions from glucocorticoids and thyroid hormone (Siebler et al., 2001). At the growth plate, all three factors converge via locally acting IGF-1, which binds to IGF-1 receptors on chondrocytes, stimulating their proliferation. One possibility is that fetal programming may occur via the growth hormone neuro-endocrine axis, where alterations could potentially affect growth plate structure and function, possibly through local availability of IGF-1. However, IGF-1 mainly influences cell proliferation. Although bone growth is not simply restricted to the control of skeletal cell proliferation; the main parameters contributing to linear growth are matrix synthesis and cellular enlargement during hypertrophy (Hunziker and Schenk, 1989; Breur et al., 1991; Kuhn et al., 1996; Farnum et al., 2002). This has been studied in the proximal tibial growth plate of 4-week old rats. At this age the animal is in its pubertal growth spurt. In the tibia only $9 \%$ of growth is contributed by proliferation, whereas $32 \%$ is due to matrix synthesis and $59 \%$ as a consequence of chondrocyte enlargement (Wilsman et al., 1996). Hence, to understand the possible effects of programming on the bone development requires an understanding of the regulation of longitudinal growth that occurs at the growth plate.

In larger mammals, the growth plates close at skeletal maturity and hence longitudinal growth ceases. However, smaller mammals such as rats and mice maintain a growth plate into old age (Roach et al., 2003). In rats the rate of growth increases during the first 1 to 5 weeks after birth and subsequently declines until skeletal maturity at approximately 11.5 to 13 weeks of age (Kember, 1973; Hunziker and Schenk, 1989). Therefore, bones continue to grow at a reduced rate until around 26 weeks of age, after which growth virtually ceases and the growth plate height is fixed. Therefore, after 5 weeks of age, the rate of growth starts to decline and the height of the growth plate continually decreases (Roach et al., 2003). During this time period the greater the height of the growth plate, the lower the level of skeletal maturity. It should be noted that changes do still occur within the growth plate structure after 26 weeks of age (Roach et al., 2003) with areas where chondrocyte death occurs resulting in large acellular areas, or 'core' cartilage is resorbed and replaced by bone, or there is direct bone formation by former growth plate chondrocytes.

Growth in the developing offspring also has a significant skeletal stem cell cellular component. Bone formation and subsequent bone growth in the developing offspring depend on the differentiation of bone marrow mesenchymal stem cells into cells of the osteogenic lineage (Tare et al., 2008; Tare et al., 2010). Therefore, differences in bone quality are likely to be as a consequence of altered regulation of skeletal/mesenchymal stem cell activity. It is this exquisite temporal and spatial process of bone formation that provides a potential mechanism for the programming of the skeletal growth trajectory by dietary protein restriction during intrauterine life.

To help dissect out the mechanisms involved in skeletal programming and the role of nutrition, in general two methods are applied; an isocaloric maternal low protein diet or utilisation of varying degrees of global reduction in nutrition - each will be detailed below. However, it is important to note, that data is now emerging that, even if an isocaloric low protein diet does not affect birth weight significantly in the first generation, an isocaloric low protein diet may affect birth weight in subsequent generations (Stewart et al., 1975). Thus it is clear that not only are the offspring of the first generation subject to influence but also the $\mathrm{F}_{2}$ gametes in the female are affected.

\section{Fetal Growth and Nutrition}

A variety of factors is critical for optimum fetal growth such as oxygen, growth factors and protein. Thus, for example, insulin is an important fetal growth hormone and through most of fetal life, amino acids, rather than glucose, determine insulin secretion by $\beta$-pancreatic cells (Hales and Barker, 1992). Furthermore, a maternal low protein diet during pregnancy affects islet cells as well as insulinsensitive organs like liver, muscle, kidney and brain in the offspring (Langley-Evans, 2000). IGF-1 has been shown to play a role in fetal growth with both maternal and fetal IGF-1 regulated by nutrition (Gluckman et al., 1996). In a rat model, when mothers were fed $30 \%$ of ad libitum diet, maternal IGF-1 levels were observed to be reduced throughout pregnancy and, critically, late gestation fetuses and placental weights were significantly reduced (Woodall et al., 1996). In a guinea pig model, mothers were fed a 70 
$\%$ of ad libitum diet and fetal measurements taken on day 40 or a 70 day gestation (Sohlström et al., 2001). Maternal IGF-1 and IGF-2 levels were significantly reduced, total fetal mass per dam was reduced by $29 \%$ and placental mass by $23 \%$. Support for a role of IGF-1 has also been observed in large animal models of development, Thus, in sheep, raising IGF-1 levels in pregnant ewes resulted in increased uptake of amino acids and glucose by the placenta and enhanced glucose delivery to the fetus (Harding et al., 1994), whereas in growth restricted fetuses, raising IGF-1 levels produces different effects suggesting altered sensitivity to IGF-1 depending on the cause, duration and severity of growth retardation (Jensen et al., 1999).

In the last 10 years, work from the Karsenty Laboratory has shown that bone is an important endocrine organ (Ducy et al., 2000; Takeda et al., 2002; Hinoi et al., 2009; Ferron et al., 2010), with leptin, insulin, and osteocalcin all playing important roles in both bone development and energy metabolism. Hence, bone is not simply an organ that is affected by other systems, but bone can also influence energy metabolism and male fertility (in mice) (Oury et al., 2011), via the action of osteocalcin on testosterone production.

Therefore, there is compelling emerging evidence that modulation of bone formation and bone growth in the developing offspring as a consequence of altered bone marrow mesenchymal stem cells fate and function are likely to affect bone quality and provide candidate mechanisms for the programming of the skeletal growth trajectory by dietary protein restriction during intrauterine life. Furthermore, whilst bone growth and development is influenced by hormones from other systems, there is evidence that bone itself may control production of these hormones, implying a role for bone in fetal growth.

\section{Changes to Bone Development in Utero}

There is now substantial accumulating evidence recording alterations in bone development during gestation or shortly after birth. Hastings-Roberts and Zeman (Hastings-Roberts and Zeman, 1977) used a severe protein restriction rat model comprising a diet of $24 \%$ casein (control) or 4 $\%$ casein (deficient), both fed ad libitum through day 20 of gestation, or $4 \%$ casein fed ad libitum until day 16 of gestation followed by $24 \%$ casein ad libitum to day 20 (supplemented). Fetuses of protein-deficient dams had the fewest number of ossification centres compared to controls. The number of ossification centres in the fetuses in the supplemented group was also observed to be significantly reduced in comparison to controls. However, protein supplementation resulted in a significant improvement in fetal bone development over the deficient group as evidenced by an increase in the number of visible ossification sites.

Fleeman's group (Fleeman et al., 2005) used a different dietary manipulation and studied the effect of total nutrient restriction in pregnant rat dams. There were no skeletal malformations associated with nutrient restriction or fetal weight reduction in gestational day 21 fetuses. There were reductions in ossification levels associated with the lowest nutrient level (62.5\% reduction). Interestingly, although maternal and fetal body weights were significantly reduced in the other feed-restricted groups $(25 \%$ and 50 $\%$ reduction), there was no evidence of delayed skeletal ossification in these groups. Whereas Cappon et al. (Cappon et al., 2005) found that using a food restriction of $50 \%$ or more in the rabbit from gestational days 7-19 was associated with reduced ossification of the fetal skeleton at gestational day 29, but there were no skeletal malformations associated with nutrient restriction. This may reflect differences in food utilisation efficiencies between different species.

Using a low-protein model in micro-swine, Lanham et al. found that the proximal femur from offspring 2 days before birth showed a reduced level of bone (Lanham et al., 2009). In addition, the trabeculae displayed raised density, although there were no significant differences in trabecular thickness or spacing. This structure was shown to support a lower load at failure. In contrast, in vertebra from the same animals, there were no observed differences in trabecular density or thickness, although there was an increase in trabecular spacing. The vertebral bodies were shown to support a lower load at failure. The femoral midshaft in the maternal low protein group showed no differences, whereas the midshaft of the tibia showed altered density and failed at a lower load. Furthermore, the calvaria in this group tended to be thinner, although this did not reach a significant level. Hence, cortical bone may have been less affected than trabecular bone. Interestingly, no differences were found in anthropometric measurements of the femur or vertebra.

Osgerby and colleagues (Osgerby et al., 2002), fed pregnant ewes a $30 \%$ total nutritional restriction from day 26 of gestation. Fetal samples were taken at gestational days 45,90 , and 135 . The authors reported full-term fetal IGF-I concentrations mirrored maternal levels; fetuses from undernourished mothers appearing to be higher at day 90, but were significantly lower than controls by day 135 . Insulin concentrations were not affected by maternal nutrition at day 90 , but were significantly lower at day 135 in the undernourished fetuses. IGFBP-2 levels increased significantly between days 90 and 135 in undernourished fetuses, but not in controls. Maternal diet did not significantly affect IGFBP-3 levels. From day 90 , maternal under-nutrition resulted in an increase in the length of the fetal femur and metatarsal. The trend for IGF-I to be higher in the underfed mothers in mid-gestation, and in their fetuses on day 90, may have contributed to this increase in bone length. By day 135, the fetal femur and metatarsal were of a similar length in both groups, although the fetal humerus and scapula were shorter in undernourished fetuses, suggesting differential regulation of bone growth by nutrition in a time-dependent manner.

Tatara (Tatara, 2008) studied the neonatal period by investigating the effects of treatment with $\beta$-hydroxy$\beta$-methylbutyrate (HMB) during the first 3 weeks of life in male sheep. HMB is a metabolite of the essential amino acid leucine, which appears to increase collagen deposition. Serum concentrations of bone-specific alkaline phosphatase, osteocalcin, GH and IGF-1 where raised at 21 days of age in the HMB treated group, and levels of 
the C-terminal telopeptide of type I collagen (CTX) were increased in the same group at 130 days of age. In addition, HMB improved volumetric bone mineral density and bone morphological and mechanical properties of the femur and lumbar spine, as well as increasing femoral length and femoral and vertebral volumes.

Tatara and co-workers (Tatara et al., 2007) also examined the effects of HMB treatment on pregnant pig sows during the two last weeks of gestation. HMB increased serum levels of GH, IGF-1 and bone alkaline phosphatase activity in the offspring. Furthermore, HMB also increased volumetric BMD of the trabecular and cortical bone of the femur in the offspring at 6 months of age. Overall, HMB increased the maximum elastic strength and ultimate strength of the femur. The authors suggested that these effects, together with the increased level of GH and IGF-1 in the newborns, indicated involvement of improved somatotrophic axis function in prenatal programming of skeletal development in pigs.

Thus to summarise, using a variety of different animal models and dietary challenges, these results show that maternal dietary restriction during pregnancy and shortly after birth can directly alter bone ossification in the growing fetus apparently by altering fetal serum IGF-1 and IGF binding protein function as indicated by modified expression.

\section{Long-term Changes to Bone Growth}

While there is a wealth of data on the effects of maternal diet on bone development in utero, to determine if maternal diet has a permanent effect on bone development, growth, and maintenance, longitudinal, and thus long-term, studies of animals into adulthood and old age are a necessity.

Langley-Evans developed the low-protein rat model over 15 years ago (Langley and Jackson, 1994), yet it still remains one of the most widely used animal models to examine the role of maternal nutritional deficiencies on the development of the offspring. In brief, pregnant rat dams are fed a diet containing $180 \mathrm{~g}$ casein $/ \mathrm{kg}$ (control diet) or $90 \mathrm{~g}$ casein $/ \mathrm{kg}$ (low-protein diet). The low protein diet is balanced in energy content by the addition of carbohydrates. Animals are provided with free access to water and food at all times. On the day of birth, mothers are switched to a standard laboratory chow and all litters reduced to a maximum of 8 pups. The nutritional composition of the low-protein fed mother's milk has been recorded to return to that of control mothers within 24 hours of transfer to the standard laboratory chow. Thus, differences in pup phenotype can be attributed directly the protein insufficiency in utero. Using this model, pups have been shown to develop functional changes in adulthood, including hypertension, impaired renal function and immune response, cardiovascular disease, and altered lifespan (Langley-Evans et al., 1996; Langley-Evans, 1999; Langley-Evans et al., 1999a; Langley-Evans et al., 1999b; Aihie et al., 2001).

Studies by Mehta and colleagues in 2002 using the Langley-Evans rat model together with dual-energy X-ray absorptiometry (DXA) analysis, demonstrated the mean bone area of the low-protein group adult offspring to be $10 \%$ lower than that of the control offspring (Mehta et al., 2002). A similar magnitude in difference was observed for whole body bone mineral content (BMC). However, there were no differences between control and low protein groups in whole body bone mineral density (BMD). There was a significant association between whole body fat mass and whole body BMC. Adjusting for differences in fat mass eliminated the significant effect of maternal diet on the BMC of the offspring. No effect was observed for whole body lean mass. The differences seen in bone area remained after adjusting for both lean and fat mass. These results indicate that a maternal low-protein diet culminate in a reduction in bone area and $\mathrm{BMC}$, but not $\mathrm{BMD}$, among the aged offspring, suggesting from these studies, that bone size, rather than volumetric bone density, is the principle outcome modified by maternal protein restriction. In the same study, the authors (Mehta et al., 2002) found that the height of the growth plate (both the cartilaginous and the continuous metaphyseal band of bone) in the proximal tibia at 72 weeks of age was significantly greater in male rat offspring whose mothers were protein undernourished during pregnancy. These findings suggest increased height of the growth plate in the low protein group offspring at the time when longitudinal bone growth had ceased or cessation of growth at an earlier age in the proteinundernourished rats. However, a subsequent study has shown that the low protein group male offspring display no significant differences in femur or tibia length from 4 to 75 weeks of age (Lanham et al., 2008a), suggesting the former explanation. Increased insulin levels can cause increased tibial growth plate height (Alarid et al., 1992) and aged males offspring from low protein fed mothers have been shown to have raised plasma insulin levels at 20 weeks of age (Sugden and Holness, 2002). Hence the increased growth plate height may be a consequence of higher insulin levels.

Oreffo and co-workers (Oreffo et al., 2003) using the Langley-Evans model of protein insufficiency examined the cellular mechanisms involved in the programming of bone development. Total CFU-F numbers, indicative of the colony forming efficiency of the mesenchymal stem cells and proliferation potential of these cells, at 8 weeks of age, was found to be reduced by approximately $40 \%$ in cultures from offspring whose mothers were fed a low-protein diet. The authors observed no difference at 12 weeks and a subsequent increase of $111 \%$ at 16 weeks compared to controls cultures. Examination of alkaline phosphatase-positive CFU-F number, indicative of osteogenic potential and differentiation, produced similar results. Furthermore, the authors noted the addition of osteogenic growth factors $\mathrm{GH}$, vitamin D, and IGF-1, under the conditions examined, was insufficient to overcome or reverse the effects of maternal dietary manipulation. In the skeletally mature control offspring (12-16 weeks of age) there was a reduction in CFU-F colony number and alkaline phosphatase specific activity, reflecting reduced osteoblast activity as skeletal growth maturity was reached. In contrast, for low-protein diet offspring, the reduced osteoblast activity/skeletal development at 8 weeks, during peak skeletal growth, and significantly increased 

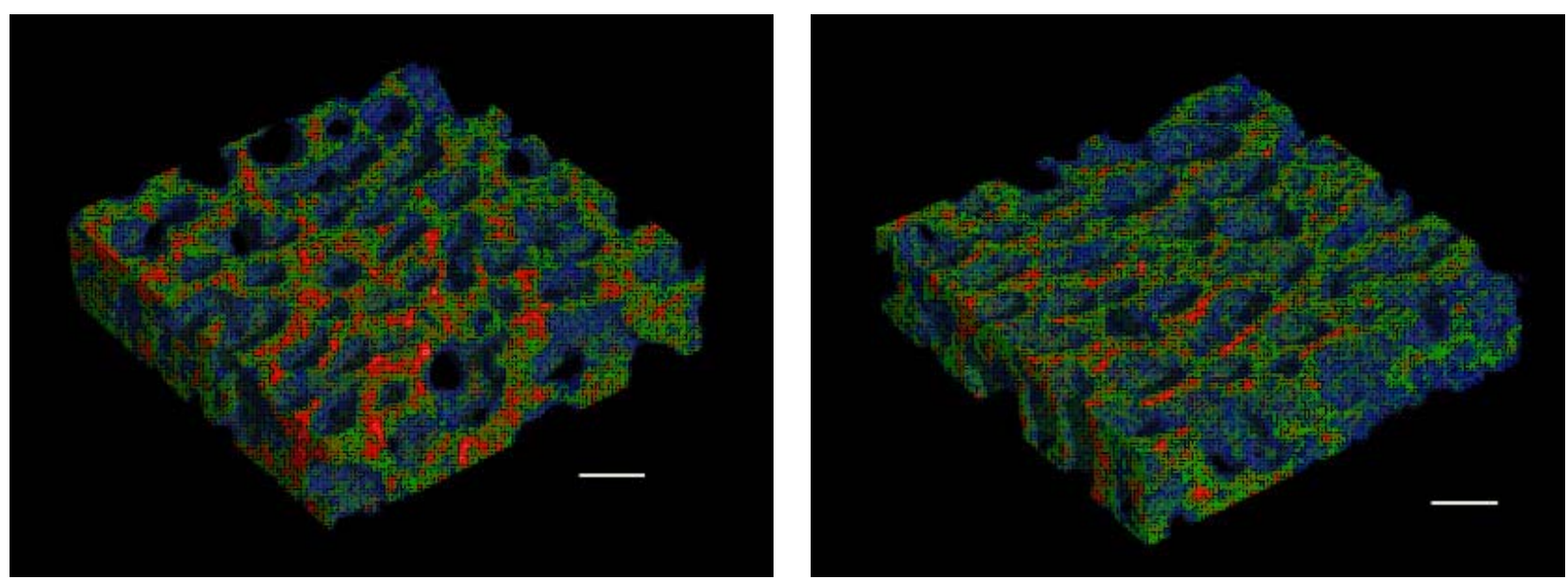

Fig. 1. False colour representative section through femoral head for 75 -week-old female offspring of rat mothers fed either control protein diet (18\%, left) or low protein $(8 \%$, right) diet during pregnancy. Images show variation in voxel density; low density bone is shown blue, medium density is shown green, and high density bone is shown red. Bar $0.5 \mathrm{~mm}$.

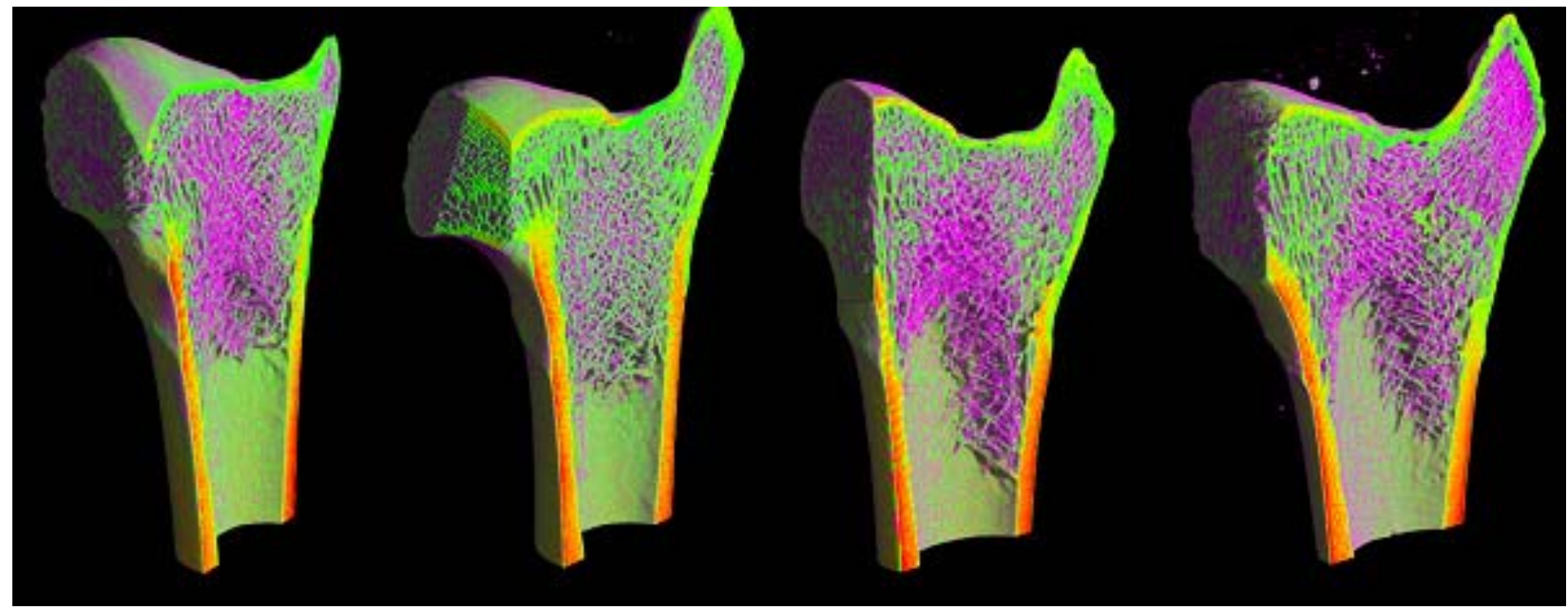

Fig. 2. Pregnant ewes were subjected to either control diet (C-) or $50 \%$ total nutrient restriction for the first 30 days of gestation (R-), male offspring were then either fed control diet (-C) or a postnatal nutrient challenge to reduce body weight to $85 \%$ of their target weight from weaning at 12 weeks to 25 weeks postnatal age (-R). Representative images show false colour section through proximal femur of male sheep for CC (left), CR (middle left), RC (middle right), or RR (right) fed offspring. Low density bone is shown magenta, medium density is shown green, and high density bone is shown yellow/orange.

osteoblast activity at 12 and 16 weeks may be attributable to intrauterine programming, with subsequent "catch-up" skeletal growth occurring at 12 and 16 weeks. However, sex differences were not reported, and in a similar study only males displayed catch-up growth (Lanham et al., 2008b).

Using the Langley-Evans model of protein insufficiency, Lanham et al. (Lanham et al., 2008b) studied markers of the osteogenic environment. Serum IGF-1 levels were significantly lower in female low-protein group offspring at 4 weeks of age and serum osteocalcin was significantly higher at 4 weeks of age in male and female offspring from mothers maintained on a low-protein diet, whereas serum $25-\mathrm{OH}$ vitamin D was significantly lower in low-protein group males at 8,12 , and 20 weeks of age. The increased levels of osteocalcin, a specific marker for osteoblast activity (Brown et al., 1984), seen in the low-protein diet group at 4 weeks of age suggested increased osteoblast activity and increased bone formation in these animals compared to controls.

Lanham et al. (Lanham et al., 2008a), used the LangleyEvans model together with micro-CT, to analyse bone structure of 75 week old female offspring from mothers fed a low-protein diet during pregnancy. The femoral heads were found to have thinner, less dense trabeculae (Fig. 1), while the femoral neck bone displayed closer packed trabeculae. Furthermore, the vertebrae had thicker, denser trabeculae, and the midshaft tibiae displayed greater dense cortical bone. Strength testing demonstrated that the femoral heads and midshaft tibiae to be structurally weaker, whereas the femoral necks and vertebrae were found to be structurally stronger. These studies provided 
the first direct evidence that maternal nutrition could alter the microscopic bone architecture and structure of the aged offspring. Interestingly, there were site-specific differences in the effects of the maternal nutrition, as the femoral head and midshaft tibia showed alterations characteristic of osteoporosis, whereas the femoral neck and vertebra showed increased bone strength. There were no differences in the anthropometric measurements of the bones. Interestingly, there appeared to be sex-specific differences in the effect of the maternal low-protein diet. Thus, when 75 week old male offspring were tested, no differences were found in structure or density in the femoral head, femoral neck, midshaft femur, midshaft tibia, or vertebra between controls and the low-protein group offspring (Lanham, Cooper, Oreffo unpublished data).

Romano et al. (Romano et al., 2009) used a bilateral uterine vessel ligation to induce a fetal total nutrient restriction in rat offspring. The authors noted that at 6 months of age, in comparison to controls, the restricted offspring had reduced $\mathrm{BMC}$, reduced anthropometric measurements and reduced bone strength. These parameters could be restored to levels seen in controls if the restricted offspring was cross-fostered onto a control mother. However, this was only observed with female offspring and not male offspring, again suggesting sex differences in the long-term influence of maternal diet. In addition, supplementation with a high calcium diet from adolescence increased adult cortical bone density in low birth weight males and females, and normal weight females. However, calcium supplementation was not sufficient to rescue the bone dimension and strength deficits which were programmed in utero, suggesting that the early life environment is critical for bone programming (Romano et al., 2010).

Over twenty five years ago Schrader et al. (Shrader and Zeman, 1973) examined the male offspring from rat dams fed either a control diet (24\% casein) or a proteindeprived diet (6\% casein) during pregnancy. Offspring were reared in foster litters of 10 (large litters) or four (small litters). Body weight was significantly reduced in the large control litters and both protein-deprived litter sets. However, the protein-deprived small litter set had a similar mass to the large control litter indicating there was a compensatory change in mass from augmentation of the food supply. Only the protein-deficient offspring displayed a delay in ossification that was not altered between the large and small litters suggesting this had been "set" by the maternal diet. From birth to 65 days of age, the rate of bone elongation and weight gain of control male pups exceeded that of deficient male pups. However, from 65 to 90 days, the tibial elongation rate was the same in the two groups while body weight gain decreased at a faster rate in controls than in deficient young.

Using a sheep model, Lanham et al. (Lanham et al., 2005) used a maternal $50 \%$ total nutrient restriction for the first 30 days of gestation, a postnatal nutrient challenge to reduce body weight to $85 \%$ of their target weight from weaning at 12 weeks to 25 weeks postnatal age, or both. Bone measurements were taken when the male offspring reached 2.5 years of age. Anthropometric measurements of femurs showed no significant differences between the groups, however all the measurements showed a trend compared to controls for the mean to be lower in the postnatal challenge group, higher in the gestation/postnatal challenge group, and similar in the gestation challenge group. When the femur was analysed by pQCT, again, no significant differences were observed between the groups, although the same trends persisted, this time with cortical and trabecular BMD, BMC, cortical thickness and trabecular area. These trends remained after normalising to body mass. Examination of vertebrae using DXA showed BMD in the gestation challenge group was significantly lower than controls, whereas the gestation/postnatal challenge group was similar to controls. No differences were found in BMC. Significance was not observed when results were normalised to mass. No significant differences were found with vertebral BMD or BMC if $50 \%$ maternal nutrient restriction was applied for 30 days before conception.

These various studies clearly demonstrate that a maternal dietary restriction during pregnancy affect bone development with effects on the bone of the growing juvenile and long-term effects in the adult offspring.

\section{Effects of dietary excess on bone growth}

Nutritional challenge does not necessitate a lack of nutrients, but may also be due to increased nutrients. Given the current obesity epidemic in the western and developing world, lessons from increased nutrient intake on skeletal development may prove informative. Driscoll et al. (Driscoll et al., 1990) studied the effect of a maternal high protein diet (40\% protein, controls $20 \%$ protein), from day 7 of gestation, on the mandibles of the newborn rat. The authors showed that the alkaline phosphatase and acid phosphatase activity from the high-protein diet group was lower than controls. Protein levels in the mandibles were higher in the high-protein diet groups, although there were no differences in the mass of the mandibles.

Lanham et al. (Lanham et al., 2010) examined the bone development in a mouse model of high fat intake, during pregnancy and/or for the offspring. Offspring were analysed at 30 weeks of age. Both males and females in the two high fat offspring groups (offspring high fat diet with maternal control or maternal high fat diet) showed increased fat deposition in the distal femur, with the maternal high fat with offspring high fat diet group demonstrating the greatest level of deposition. The male offspring were less affected than the female offspring. Male high fat fed offspring, regardless of maternal diet, showed increased mass and reduced femur length. High fat fed males from high fat fed mothers also showed an increased load at fracture of the midshaft, likely due to the increased cross-sectional area seen at this point in this group. High fat fed female offspring from control mothers displayed increased mass, increased bone volume, and increased cross-sectional area of the midshaft. In contrast, high fat fed female offspring from high fat fed mothers displaying increased mass (although not to the extent of the maternal control diet/offspring high fat diet females), showed a reduced femur length, and increased cross-sectional area of the midshaft (Fig. 3). These data 

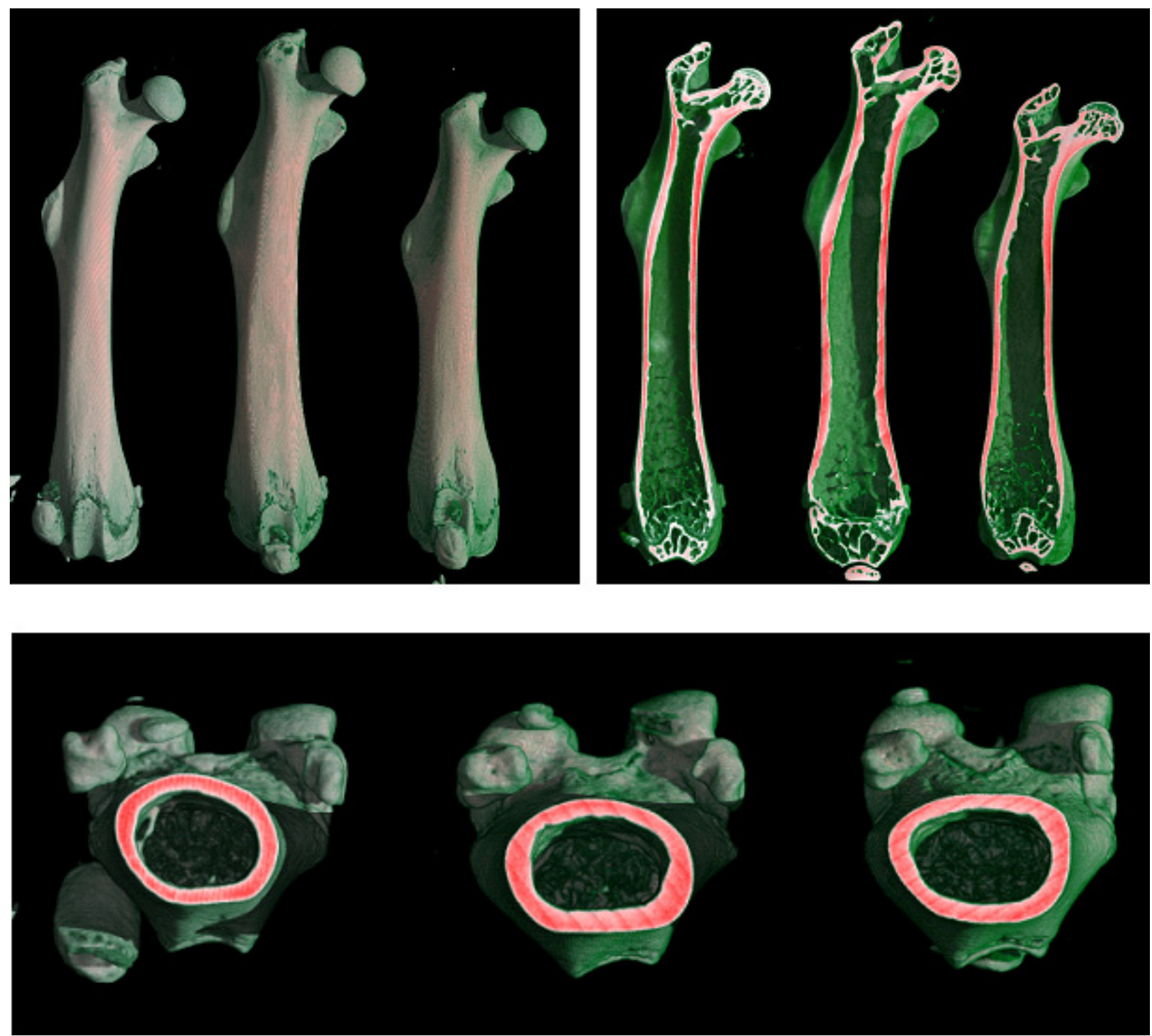

Fig. 3. False colour representations of femur from high fat fed female mouse offspring aged 30 weeks. For all images, left femur is control animal, middle is high fat fed offspring from control fed mother (C-HF), and right is high fat fed offspring from high fat fed mother (HF-HF). Top left image shows reduced femur length in HF-HF group compared to control and $\mathrm{C}-\mathrm{HF}$ groups. Top right image shows internal view of the same samples. Bottom image shows increased cross-sectional area at the midshaft in the C-HF and HF-HF groups compared to controls.

demonstrate that the maternal control diet/offspring high fat diet group increased the cross-sectional area of the femur to withstand the greater mass of the animal. Interestingly, as other bone parameters were maintained (femur length and trabecular thickness), this indicated an increase in the total amount of bone present in the femur. In contrast, the maternal high fat diet/offspring high fat diet females had similar trabecular indices to those seen in controls (Fig. $3)$. The data indicate a reduction in femur length in order to supply bone material to increase the cross-sectional area of the midshaft, to adapt with the extra mass of the animal. This suggests programming of the total amount of bone, and re-modelling to redistribute the bone to cope with an increase in mass of the animal. However, these maternal high fat diet/offspring high fat diet females failed to increase in mass to a level equal to that of the maternal control diet/offspring high fat diet group as was observed in the male offspring. Thus in summary, it would appear a maternal high fat diet altered the basal metabolic activity of the female offspring.

These data demonstrate that excess nutrients during pregnancy can affect the bones of the offspring. By inducing excess deposition or storage of nutrients, it can lead to permanent alterations of bone development and structure. As detailed below, the key will be to correlate some of these observations with human data and to begin to unravel potential mechanisms.

\section{Mechanisms of action}

Given that maternal diet appears to be able to programme bone growth in the offspring independently of offspring DNA sequence, an epigenetic mechanism would appear an important area for investigation. Epigenetic modification consists of three distinct, but closely interacting processes; 
(i) DNA methylation of carbon 5 of cytosine residues in CpG nucleotides, (ii) histone modifications, in particular acetylation and methylation on specific lysines, and (iii) microRNAs (miRNA) (Jaenisch and Bird, 2003; Iorio et al., 2010). Conformationally relaxed chromatin indicates a transcriptionally active region displaying hypomethylated DNA, acetylation of histones $\mathrm{H} 3$ and $\mathrm{H} 4$, as well as methylation of $\mathrm{H} 3$ at lysine 4 (H3 K4). Compact chromatin is transcriptionally silent, hypermethylated, and bound to non-acetylated histones with methylation at H3 K9 and K27 (Feinberg, 2007; Gan et al., 2007). Methylation of DNA occurs via the addition of methyl groups to 5 '-cytosines next to guanines (CpG sites) by DNA methyltransferases (Dnmts) (Pradhan and Esteve, 2003; Klose and Bird, 2006). These methyl groups are primarily supplied from serine. Methylated $\mathrm{CpG}$ sites attract methyl-binding proteins, which are associated with histone deacetylases and histone methyl transferases (Fujita et al., 2003; Fuks et al., 2003), thus there is a reciprocal relationship between DNA methylation and histone modifications. However, DNA methylation is important for maintaining silencing in the long-term (Bird, 2002; Reik, 2007), since DNA methylation patterns are generally stable in somatic cells throughout adult life (Berger, 2007). During DNA replication, the methylation pattern is rapidly reproduced on the new DNA by the methyl transferase DNMT1, which associates with proliferating-cell nuclear antigen and tracks with DNA polymerase (Newell-Price et al., 2000; Attwood et al., 2002). The histone code can then be re-established after cell division by various mechanisms involving methyl binding domains (MBDs) and DNMTs, which can interact with histone methyltransferases and histone deacetylases. Although a number of studies have concentrated on $\mathrm{CpG}$ methylation, there is evidence that non-CpG methylation may also be important (Fuso et al., 2010).

Methylation is affected by dietary factors, in particular by levels of methyl-donors such as folic acid (Jhaveri et al., 2001; Van den Veyver, 2002; Davis and Uthus, 2004). In addition, the DNA methylation pattern for a specific gene varies between cell types. In general, cells expressing the gene will have hypomethylated promoters, whereas hypermethylation of the promoter silences the gene (Chan et al., 2000; Attwood et al., 2002; Salozhin et al., 2005). The DNA methylation status is heritable, i.e. the cell typespecific gene expression repertoire is stably transmitted to daughter cells. However, if changes occur, for example as a consequence of intrauterine protein deficiency, then the aberrant methylation pattern will also be transmitted to daughter cells, which may result in either abnormal expression of silenced genes or suppression of normally expressed genes. Studies using the agouti mouse (Wolff et al., 1998) elegantly demonstrate this principle. Yellow (Avy/a) agouti mice are hypomethylated. However, feeding pregnant black a/a dams methyl-supplemented diets resulted in increased methylation and increased silencing of agouti expression in their offspring, as shown by increased agouti/black mottling in the direction of the pseudoagouti phenotype. The epigenetic phenotypes were maternally heritable and, critically, shown to be modulated by maternal diet, thereby adding evidence to the importance of maternal nutrition on the health and longevity of their offspring.
In our rodent studies (unpublished data), we have cultured bone marrow cells under osteogenic conditions from newborn offspring and searched for genes that were over- or under-expressed in the offspring from low protein fed mothers. We have identified 184 genes (17 over and 167 under-expressed) in males and 483 (213 over and 270 under-expressed) in female offspring. Thus, a maternal protein restriction caused sex-specific alterations in gene expression. Preliminary analyses of the DNA methylation status of genes important in bone development, found over-expression of a candidate gene was associated with decreased methylation of the promoter in $100 \%$ of overexpressing samples, but only $43 \%$ of under-expressing samples $(p<0.05)$. We also found loss of DNA methylation in the 3' UTR region of the gene (100\% of low protein offspring samples versus $0 \%$ of control samples, $p=$ 0.001 ), which is a potential binding region for microRNAs and may also be involved in the epigenetic regulation of genes. These observations provide further evidence of the influence of maternal diet on offspring bone structure and development.

\section{Animal models - relevance to human biology}

A number of non-communicable diseases of adult life have been identified as having a developmental origin, including, coronary heart disease, stroke, type 2 diabetes mellitus, adiposity, and the metabolic syndrome (reviewed in Gluckman et al., 2008). The authors show that currently identified genetic markers only account for a small proportion of the variation in bone mass and risk of fracture of individuals. Data from animal studies are particularly interesting when extrapolated to data from human studies such as the Saskatchewan bone mineral accrual study (Bailey et al., 1999) and the Finnish cohort studies (Cooper et al., 2001). These studies show that maturational delay may increase the risk of hip fracture through altered accrual of bone density and disproportionate bone growth. In addition, studies have shown that human intrauterine growth retarded babies are capable of demonstrating "catch-up" growth for various anthropometric parameters (Villar et al., 1982; Villar et al., 1984). Furthermore, infants malnourished in utero had a significant retardation in skeletal maturity at birth, when compared to controls (Walther et al., 1981). However, at 2.5-3.5 years of age, the average skeletal maturity of the intrauterine malnourished children approached that of controls, demonstrating "catchup" in skeletal growth during infancy.

Yin et al. (Yin et al., 2009) studied maternal dietary intake in the third trimester of pregnancy and bone mass in the male and female offspring at age 16. The authors concluded that maternal milk intake was positively associated with offspring bone mineral density at age 16, whereas maternal fat intake showed a negative association. The authors concluded that maternal diet influenced peak bone mass in the offspring possibly through programming bone responses.

A recent meta-analysis (Baird et al., 2011) looked at birthweight or weight at 1 year of age to bone mineral content, bone mineral density or osteoporotic fracture in adults aged 18 years and over. From over 4000 abstracts, 14 worldwide studies fitted their inclusion criteria. From 
these, the authors concluded there was a consistent positive association between birth weight and adult bone mineral content (BMC) at the lumbar spine and hip; the association being stronger in women. However, birth weight was not a predictor of areal bone mineral density of the lumbar spine and hip. In addition, there was less consistent evidence about the relationship between birth weight and bone mass at the neck of femur, radius or ulna (Baird et al., 2010a).

\section{Summary}

A wealth of data is now emerging indicating that maternal diet can greatly affect the size, structure and strength of the bones in the offspring. Abnormalities of skeletal development are initiated in early life, potentially through impaired IGF-1 activity, which would favour downregulation of bone deposition. Thus, changes in skeletal growth and structure following poor nutrition in pregnancy may lead to reduced bone mass in later life. This could potentially results in osteoporotic fracture in humans. However, as indicated above, caution must be applied before extrapolating animal findings to human populations.

It is clear, animal models of maternal dietary insufficiency and excess provide useful models to study skeletal programming in utero. The challenge for the next decade will be to identify the mechanisms of programming of bone growth and to enhance our understanding of the factors influencing bone development, adult bone structure and peak bone mass. Advances in our understanding of the developmental origins of bone and bone diseases will undoubtedly provide a step change in clinical treatment and reduction in the burden of osteoporosis for an increasing ageing population.

\section{Acknowledgements}

Research by the authors is supported by the Biotechnology and Biological Sciences Research Council, Research into Ageing and Medical Research Council.

\section{Disclosures}

The authors state that they have no conflicts of interest.

\section{References}

Aihie S, Dunn R, Langley-Evans SC, Cooper C (2001) Prenatal exposure to a maternal low protein diet shortens life span in rats. Gerontology 47: 9-14.

Alarid ET, Schlechter NL, Russell SM, Nicoll CS (1992) Evidence suggesting that insulin-like growth factor-I is necessary for the trophic effects of insulin on cartilage growth in vivo. Endocrinology 130: 2305-2309.

Attwood JT, Yung RL, Richardson BC (2002) DNA methylation and the regulation of gene transcription. Cellular and Molecular Life Sciences (CMLS) 59: 241-257.

Bailey DA, McKay HA, Mirwald RL, Crocker PR,
Faulkner RA (1999) A six-year longitudinal study of the relationship of physical activity to bone mineral accrual in growing children: the university of Saskatchewan bone mineral accrual study. J Bone Miner Res 14: 1672-1679.

Baird J, Kurshid M, Kim M, Harvey N, Dennison E, Cooper C (2011) Does birthweight predict bone mass in adulthood? A systematic review and meta-analysis. Osteoporos Int 22: 1323-1334.

Barker DJP (1992) The fetal origins of diseases of old age. Eur J Clin Nutr 46 Suppl 3: S3-S9.

Berger SL (2007) The complex language of chromatin regulation during transcription. Nature 447: 407-412.

Bird A (2002) DNA methylation patterns and epigenetic memory. Genes Dev 16: 6-21.

Breur GJ, Vanenkevort BA, Farnum CE, Wilsman NJ (1991) Linear relationship between the volume of hypertrophic chondrocytes and the rate of longitudinal bone-growth in growth plates. J Orthopaed Res 9: 348-359.

Brown JP, Delmas PD, Malaval L, Edouard C, Chapuy MC, Meunier PJ (1984) Serum bone Gla-protein: a specific marker for bone formation in postmenopausal osteoporosis. Lancet 1: 1091-1093.

Cappon GD, Fleeman TL, Chapin RE, Hurtt ME (2005) Effects of feed restriction during organogenesis on embryo-fetal development in rabbit. Birth Defects Res B Dev Reprod Toxicol 74: 424-430.

Chan MF, Liang G, Jones PA (2000) Relationship between transcription and DNA methylation. Curr Top Microbiol Immunol 249: 75-86.

Cooper C, Cawley M, Bhalla A, Egger P, Ring F, Morton L, Barker DJP (1995) Childhood growth, physical activity, and peak bone mass in women. J Bone Mineral Res 10: 940-947.

Cooper C, Fall C, Egger P, Hobbs R, Eastell R, Barker DJP (1997) Growth in infancy and bone mass in later life. Ann Rheum Dis 56: 17-21.

Cooper C, Eriksson JG, Forsen T, Osmond C, Tuomilehto J, Barker DJ (2001) Maternal height, childhood growth and risk of hip fracture in later life: a longitudinal study. Osteoporos Int 12: 623-629.

Cooper C, Javaid MK, Taylor P, Walker-Bone K, Dennison EM, Arden N (2002) The fetal origins of osteoporotic fracture. Calcif Tissue Int 70: 391-394.

Davis CD, Uthus EO (2004) DNA Methylation, cancer susceptibility, and nutrient interactions. Exp Biol Med 229: 988-995.

Driscoll PG, Joseph F, Nakamoto T (1990) Prenatal effects of maternal caffeine intake and dietary high protein on mandibular development in fetal rats. Br J Nutr 63: 285-292.

Ducy P, Amling M, Takeda S, Priemel M, Schilling AF, Beil FT, Shen J, Vinson C, Rueger JM, Karsenty G (2000) Leptin inhibits bone formation through a hypothalamic relay: a central control of bone mass. Cell 100: 197-207.

Fall C, Hindmarsh P, Dennison EM, Kellingray S, Barker DJP, Cooper C (1998) Programming of growth hormone secretion and bone mineral density in elderly men: a hypothesis. J Clin Endocrinol Metab 83: 135-139.

Farnum CE, Lee R, O’Hara K, Urban JPG (2002) Volume increase in growth plate chondrocytes during hypertrophy: The contribution of organic osmolytes. Bone 
30: 574-581.

Feinberg AP (2007) Phenotypic plasticity and the epigenetics of human disease. Nature 447: 433-440.

Ferron M, Wei J, Yoshizawa T, Del Fattore A, DePinho RA, Teti A, Ducy P, Karsenty G (2010) Insulin signaling in osteoblasts integrates bone remodeling and energy metabolism. Cell 142: 296-308.

Fleeman TL, Cappon GD, Chapin RE, Hurtt ME (2005) Effects of feed restriction during organogenesis on embryo-fetal development in the rat. Birth Defects Res B Dev Reprod Toxicol 74: 442-449.

Fujita N, Watanabe S, Ichimura T, Tsuruzoe S, Shinkai Y, Tachibana M, Chiba T, Nakao M (2003) Methyl-CpG binding domain 1 (MBD1) interacts with the Suv39h1-HP1 heterochromatic complex for DNA methylation-based transcriptional repression. J Biol Chem 278: 24132-24138.

Fuks F, Hurd PJ, Wolf D, Nan X, Bird AP, Kouzarides $\mathrm{T}$ (2003) The methyl-CpG-binding protein MeCP2 links DNA methylation to histone methylation. J Biol Chem 278: 4035-4040.

Fuso A, Ferraguti G, Grandoni F, Ruggeri R, Scarpa S, Strom R, Lucarelli M (2010) Early demethylation of non-CpG, CpC-rich, elements in the myogenin 5'-flanking region: a priming effect on the spreading of active demethylation. Cell Cycle 9: 3965-3976.

Gan Q, Yoshida T, McDonald OG, Owens GK (2007) Concise review: Epigenetic mechanisms contribute to pluripotency and cell lineage determination of embryonic stem cells. Stem Cells 25: 2-9.

Gluckman P, Hanson MA (2006) Developmental origins of health and disease. Cambridge University Press, Cambridge.

Gluckman PD, Cutfield W, Harding JE, Milner D, Jensen E, Woodhall S, Gallaher B, Bauer M, Breier BH (1996) Metabolic consequences of intrauterine growth retardation. Acta Paediatr 85: 3-6.

Gluckman PD, Cutfield W, Hofman P, Hanson MA (2005) The fetal, neonatal, and infant environments-the long-term consequences for disease risk. Early Hum Dev 81: 51-59.

Gluckman PD, Hanson MA (2004) Living with the past: evolution, development, and patterns of disease. Science 305: 1733-1736.

Gluckman PD, Hanson MA, Cooper C, Thornburg KL (2008) Effect of in utero and early-life conditions on adult health and disease. N Engl J Med 359: 61-73.

Hales CN, Barker DJ (1992) Type 2 (non-insulindependent) diabetes mellitus: the thrifty phenotype hypothesis. Diabetologia 35: 595-601.

Harding JE, Liu L, Evans PC, Gluckman PD (1994) Insulin-like growth factor 1 alters feto-placental protein and carbohydrate metabolism in fetal sheep. Endocrinology 134: 1509-1514.

Hastings-Roberts MM, Zeman FJ (1977) Effects of protein deficiency, pair-feeding, or diet supplementation on maternal, fetal and placental growth in rats. J Nutr 107: 973-982.

Hinoi E, Gao N, Jung DY, Yadav V, Yoshizawa T, Kajimura D, Myers J, Chua J, Wang Q, Kim JK, Kaestner KH, Karsenty G (2009) An osteoblast-dependent mechanism contributes to the leptin regulation of insulin secretion. Ann N Y Acad Sci 1173: E20-E30.

Hunziker EB, Schenk RK (1989) Physiological mechanisms adopted by chondrocytes in regulating longitudinal bone growth in rats. J Physiol (Lond) 414: 55-71.

Iorio MV, Piovan C, Croce CM (2010) Interplay between microRNAs and the epigenetic machinery: an intricate network. Biochim Biophys Acta 1799: 694-701.

Jaenisch R, Bird A (2003) Epigenetic regulation of gene expression: how the genome integrates intrinsic and environmental signals. Nat Genet 33 Suppl: 245-254.

Jensen EC, Harding JE, Bauer MK, Gluckman PD (1999) Metabolic effects of IGF-I in the growth retarded fetal sheep. J Endocrinol 161: 485-494.

Jhaveri MS, Wagner C, Trepel JB (2001) Impact of extracellular folate levels on global gene expression. Mol Pharmacol 60: 1288-1295.

Jordan KM, Cooper C (2002) Epidemiology of osteoporosis. Best Pract Res Clin Rheumatol 16: 795-806.

Kember NF (1973) Aspects of the maturation process in growth cartilage in the rat tibia. Clin Orthop 95: 288-294.

Klose RJ, Bird AP (2006) Genomic DNA methylation: the mark and its mediators. Trends Biochem Sci 31: 89-97.

Kuhn JL, Delacy JH, Leenellett EE (1996) Relationship between bone growth rate and hypertrophic chondrocyte volume in New Zealand white rabbits of varying ages. J Orthop Res 14: 706-711.

Langley SC, Jackson AA (1994) Increased systolic blood-pressure in adult-rats induced by fetal exposure to maternal low-protein diets. Clin Sci 86: 217-222.

Langley-Evans SC (1999) Fetal origins of adult disease. Br J Nutr 81: 5-6.

Langley-Evans SC (2000) Critical differences between two low protein diet protocols in the programming of hypertension in the rat. Int J Food Sci Nutr 51: 11-17.

Langley-Evans SC, Sherman RC, Welham SJM, Nwagwu MO, Gardner DS, Jackson AA (1999a) Intrauterine programming of hypertension: the role of the renin-angiotensin system. Biochem Soc Trans 27: 88-93.

Langley-Evans SC, Welham SJM, Jackson AA (1999b) Fetal exposure to a maternal low protein diet impairs nephrogenesis and promotes hypertension in the rat. Life Sci 64: 965-974.

Langley-Evans SC, Gardner DS, Jackson AA (1996) Maternal protein restriction influences the programming of the rat hypothalamic-pituitary-adrenal axis. J Nutr 126: 1578-1585.

Lanham S, Roberts C, Hollingworth T, Sreekumar R, Elahi M, Cagampang F, Hanson M, Oreffo R (2010) Maternal high-fat diet: effects on offspring bone structure. Osteoporos Int 21: 1703-1714.

Lanham S, Roberts C, Perry M, Cooper C, Oreffo ROC (2008a) Intrauterine programming of bone. Part 2: Alteration of skeletal structure. Osteoporos Int 19: 157167.

Lanham SA, DuPriest E, Kupfer P, Roberts C, Cooper C, Bagby SP, Oreffo ROC (2009) Alteration of fetal bone structure by a maternal low protein diet. J Developm Origin Health Dis 1: s285.

Lanham SA, Roberts C, Burford J, Skerry TM, Taylor P, Green LR, Hanson MA, Cooper C, Oreffo ROC (2005) 
Intrauterine programming of skeletal development. Pediatr Res 58: 1045.

Lanham SA, Roberts C, Cooper C, Oreffo ROC (2008b) Intrauterine programming of bone. Part 1: Alteration of the osteogenic environment. Osteoporos Int 19: 147-156.

Mehta G, Roach HI, Langley-Evans SC, Taylor P, Reading I, Oreffo ROC, Aihie-Sayer A, Clarke NM, Cooper $C$ (2002) Intrauterine exposure to a maternal low protein diet reduces adult bone mass and alters growth plate morphology in rats. Calcif Tissue Int 71: 493-498.

Miller JP, German RZ (1999) Protein malnutrition affects the growth trajectories of the craniofacial skeleton in rats. J Nutr 129: 2061-2069.

Newell-Price J, Clark AJL, King P (2000) DNA methylation and silencing of gene expression. Trends Endocrinol Metab 11: 142-148.

Oreffo ROC, Lashbrooke B, Roach HI, Clarke NM, Cooper C (2003) Maternal protein deficiency affects mesenchymal stem cell activity in the developing offspring. Bone 33: 100-107.

Osgerby JC, Wathes DC, Howard D, Gadd TS (2002) The effect of maternal undernutrition on ovine fetal growth. J Endocrinol 173: 131-141.

Oury F, Sumara G, Sumara O, Ferron M, Chang H, Smith C, Hermo L, Suarez S, Roth B, Ducy P, Karsenty $G$ (2011) Endocrine regulation of male fertility by the skeleton. Cell 144: 796-809.

Ozaki T, Nishina H, Hanson MA, Poston L (2001) Dietary restriction in pregnant rats causes gender-related hypertension and vascular dysfunction in offspring. $\mathrm{J}$ Physiol (Lond) 530: 141-152.

Pradhan S, Esteve PO (2003) Mammalian DNA (cytosine-5) methyltransferases and their expression. Clin Immunol 109: 6-16.

Ralston SH (1998) Do genetic markers aid in risk assessment? Osteoporos Int 8 (Suppl 1): S37-S42.

Reichling TD, German RZ (2000) Bones, muscles and visceral organs of protein-malnourished rats (Rattus norvegicus) grow more slowly but for longer durations to reach normal final size. J Nutr 130: 2326-2332.

Reik W (2007) Stability and flexibility of epigenetic gene regulation in mammalian development. Nature 447: 425-432.

Roach HI, Mehta G, Oreffo ROC, Clarke NMP, Cooper C (2003) Temporal analysis of rat growth plates: cessation of growth with age despite presence of a physis. J Histochem Cytochem 51: 373-383.

Romano T, Wark JD, Owens JA, Wlodek ME (2009) Prenatal growth restriction and postnatal growth restriction followed by accelerated growth independently program reduced bone growth and strength. Bone 45: 132-141.

Romano T, Wark JD, Wlodek ME (2010) Calcium supplementation does not rescue the programmed adult bone deficits associated with perinatal growth restriction. Bone 47: 1054-1063.

Salozhin SV, Prokhorchuk EB, Georgiev GP (2005) Methylation of DNA - one of the major epigenetic markers. Biochemistry (Mosc ) 70: 525-532.

Shrader RE, Zeman FJ (1973) Skeletal development in rats as affected by maternal protein deprivation and postnatal food supply. J Nutr 103: 792-801.
Siebler T, Robson H, Shalet SM, Williams GR (2001) Glucocorticoids, thyroid hormone and growth hormone interactions: implications for the growth plate. Horm Res 56 Suppl 1: 7-12.

Sohlström A, Fernberg P, Owens JA, Owens PC (2001) Maternal nutrition affects the ability of treatment with IGF-I and IGF-II to increase growth of the placenta and fetus, in guinea pigs. Growth Horm IGF Res 11: 392-398.

Stewart RJC, Preece RF, Sheppard HG (1975) Twelve generations of marginal protein deficiency. Br J Nutr 33: 233-253.

Sugden MC, Holness MJ (2002) Gender-specific programming of insulin secretion and action. J Endocrinol 175: 757-767.

Takeda S, Elefteriou F, Levasseur R, Liu X, Zhao L, Parker KL, Armstrong D, Ducy P, Karsenty G (2002) Leptin regulates bone formation via the sympathetic nervous system. Cell 111: 305-317.

Tare RS, Kanczler J, Aarvold A, Jones AM, Dunlop DG, Oreffo RO (2010) Skeletal stem cells and bone regeneration: translational strategies from bench to clinic. Proc Inst Mech Eng H 224: 1455-1470.

Tare RS, Babister JC, Kanczler J, Oreffo ROC (2008) Skeletal stem cells: Phenotype, biology and environmental niches informing tissue regeneration. Mol Cell Endocrinol 288: $11-21$.

Tatara MR (2008) Neonatal programming of skeletal development in sheep is mediated by somatotrophic axis function. Exp Physiol 93: 763-772.

Tatara MR, Sliwa E, Krupski W (2007) Prenatal programming of skeletal development in the offspring: Effects of maternal treatment with [beta]-hydroxy-[beta]methylbutyrate (HMB) on femur properties in pigs at slaughter age. Bone 40: 1615-1622.

Van den Veyver IB (2002) Genetic effects of methylation diets. Ann Rev Nutr 22: 255-282.

Villar J, Belizan JM, Spalding J, Klein RE (1982) Postnatal growth of intrauterine growth retarded infants. Early Hum Dev 6: 265-271.

Villar J, Smeriglio V, Martorell R, Brown CH, Klein RE (1984) Heterogeneous growth and mental development of intrauterine growth-retarded infants during the first 3 years of life. Pediatrics 74: 783-791.

Walther FJ, Ramaekers LHJ, Vanengelshoven JMA (1981) Skeletal maturity at birth and at the age of 3 years of infants malnourished in utero. Early Human Developm 5: 139-143.

Widdowson EM, McCance RA (1963) The effect of finite periods of undernutrition at different ages on the composition and subsequent development of the rat. Proc Roy Soc Lond B 158: 329-342.

Wilsman NJ, Farnum CE, Green EM, Lieferman EM, Clayton MK (1996) Cell cycle analysis of proliferating zone chondrocytes in growth plates elongating at different rates. J Orthop Res 14: 562-572.

Wolff GL, Kodell RL, Moore SR, Cooney CA (1998) Maternal epigenetics and methyl supplements affect agouti gene expression in Avy/a mice. FASEB J 12: 949-957.

Woodall SM, Breier BH, Johnston BM, Gluckman PD (1996) A model of intrauterine growth retardation caused by chronic maternal undernutrition in the rat: effects on 
the somatotrophic axis and postnatal growth. J Endocrinol 150: 231-242.

Yin J, Dwyer T, Riley M, Cochrane J, Jones G (2009) The association between maternal diet during pregnancy and bone mass of the children at age 16. Eur J Clin Nutr 64: 131-137.

\section{Discussion with Reviewers}

Reviewer II: Do you think that there may be differences in the mechanism causing the programming of poor adult bone health via maternal protein restriction models and those using bilateral uterine vessel ligation as a model of uteroplacental insufficiency?

Authors: Our working hypothesis is that in utero males adapt to the nutritional environment to provide maximum growth and size and birth, whereas females adapt for maximum life expectancy. Hence, we believe the pathways involved would be the same in both nutrient situations, however, there outcomes would be expected to be different due to the proportions of each available nutrient (protein, fat, etc).
Reviewer II: Considering that maternal undernutrition is mainly now a 3rd world issue, how relevant are maternal protein restriction models to the wider community? In saying this, I would suspect that maternal overnutrition or obestiy in pregnancy may be a more important issue now affecting Western Society.

Authors: We believe there are "nutritional adaptive" pathways. In the first phase of our work - some 5 years ago, we utilised both low protein and high fat diets to validate and provide proof of concept data for the skeletal system, for this hypothesis. It has been long recognised in the cardiovascular and hypertension arena the importance of maternal nutrition modulation and the effects on offspring in later life and consequent disease risk.

What is important, and hopefully outlined in the review, is that "abnormal" nutrition either by total excess or reduction of a component (e.g., protein) can produce long term alteration of bone growth and structure - which is now only gradually gaining acceptance. Critically, the challenge facing us all is the growing epidemic of diabetes, metabolic syndrome and obesity in the western world and, critically and sadly still perhaps not fully appreciated in all quarters, the staggering rise in countries such as India and the Asian content. 